\section{Volle Asthmakontrolle auch ohne Glukokortikoide}

B ei Asthmaerkrankungen im Kindesalter handelt es sich meist um leichtes intermittierendes oder persistierendes Asthma. In internationalen Leitlinien gelten inhalative Glukokortikoide als Therapie der ersten Wahl, Studienergebnisse der letzten Jahre deuten allerdings darauf hin, dass auch mit dem Leukotrienantagonisten Montelukast eine effektive Kontrolle möglich ist.

Mithilfe einer Medline-Recherche sind jetzt die Ergebnisse der Studien der letzten zehn Jahre zusammengetragen und publiziert worden, in denen Kinder mit leichtem Asthma mit Montelukast in Monotherapie behandelt worden waren (Wahn U et al. Clin Ther 2008; 30: 1026-35). Das Ergebnis zeigt, dass eine Behandlung mit Montelukast bei Kindern mit leichtem
Asthma im Alter von zwei bis 14 Jahren - im Hinblick auf Lungenfunktion und Symptomatik - eine effektive und vorteilhafte Asthmakontrolle ermöglicht. Die einmal tägliche Gabe zeigte insbesondere bei jüngeren Kindern hinsichtlich der Anwendungsfreundlichkeit Vorteile. In verschiedenen Studien war die orale Therapie mit Montelukast mit einer signifikant höheren Therapietreue und Patientenzufriedenheit im Vergleich zu Inhalationsbehandlungen assoziiert. Darüber hinaus scheint es Patienten zu geben, die jeweils entweder nur auf inhalative Glukokortikoide oder nur auf Montelukast ansprechen. Es sollten also beide Therapieoptionen für Kinder mit Asthma zur Verfügung stehen.

Nach Informationen von MSD, Haar
Ausgezeichneter DermatikaSpezialist

Das Unternehmen Almirall Hermal hat die Goldene Tablette 2008 als beliebtestes Unternehmen im Bereich Dermatologie gewonnen. Die Auszeichnung wird jährlich vergeben und ist das Ergebnis einer repräsentativen Umfrage der Fachzeitschrift PharmaBarometer.

Der Erfolg in diesem Jahr hat einen besonderen Stellenwert, weil er belegt, dass die Dermatologen dem 1945 gegründeten Dermatika-Spezialisten Hermal auch nach der Akquisition durch das spanische Pharmaunternehmen Almirall die Treue halten.

Weitere Informationen zu Almirall Hermal bietet unter der Adresse www.almirall.de das Internet.

Nach Informationen von Almirall Hermal, Reinbek

\section{Neuer Allergenhersteller}

Seit August dieses Jahres sind die Allergenextrakte des italienischen Unternehmens Lofarma SpA auch in Deutschland verfügbar. Die deutsche Tochter Lofarma Deutschland GmbH vertreibt mit den Präparaten Lais Tropfen und Lais Tabletten das erste und einzige in Deutschland erhältliche sublinguale Allergoid (Monoid) sowie mit Lofarma Depot ein klassisches Injektionspräparat zur subkutanen Hyposensibilisierung.

Die Besonderheit von Lais liegt in seinem patentierten Herstellungsverfahren, der Carbamylierung, mit der es gelingt, die positiven Eigenschaften eines Allergoids mit einer höheren Magensaftresistenz zu verbinden. Das Ergebnis ist ein Allergenextrakt, der im Vergleich zu nativen Allergenen eine höhere Bioverfügbarkeit bietet. Somit kann auch mit vergleichsweise niedrigeren Allergendosen eine effektive Therapie erfolgen, wie die Ergebnisse zahlreicher doppelblinder und plazebokontrollierter Studien zeigen. Lais ist in Form von Sublingualtropfen oder auch -tabletten erhältlich, das Allergenportfolio deckt die häufigsten Auslöser der allergischen Rhinitis ab.

Weitere Informationen zu den Produkten können postalisch bei der Lofarma Deutschland $\mathrm{GmbH}$, Hanns-MartinSchleyer-Straße 26, 47877 Willich, oder per E-Mail an info@lofarma.de angefordert werden

Nach Informationen von Lofarma Deutschland, Willich 